\title{
The origin and fate of short-period low-mass black-hole binaries
}

\author{
L. R. Yungelson ${ }^{1,2,3,7}$, J.-P. Lasota ${ }^{3}$, G. Nelemans ${ }^{4,5}$, G. Dubus ${ }^{3,6}$, E. P. J. van den Heuvel $^{7}$, \\ J. Dewi ${ }^{5,7}$, and S. Portegies Zwart ${ }^{7,8}$
}

1 Institute of Astronomy of the Russian Academy of Sciences, 48 Pyatnitskaya Str., 119017 Moscow, Russia e-mail: lasota@iap.fr

2 Isaac Newton Institute, Moscow branch, 13 Universitetskii PR., Moscow, Russia

3 Institut d'Astrophysique de Paris, UMR 7095 CNRS, Université Pierre et Marie Curie, 98 bis Bd. Arago, 75014 Paris, France

4 Department of Astrophysics, Radboud University Nijmegen, Toernooiveld 1, 6525 ED Nijmegen, The Netherlands

5 Institute of Astronomy, University of Cambridge, Madingley Road, CB3 0HA, Cambridge, UK

${ }^{6}$ Laboratoire Leprince-Ringuet, UMR 7638 CNRS, École Polytechnique, 91128 Palaiseau, France

Astronomical Institute "Anton Pannekoek", Kruislaan 403, 1098 SJ Amsterdam, The Netherlands

8 Section Computational Science, Informatics Institute, Kruislaan 403, 1098, SJ Amsterdam, The Netherlands

Received 8 February 2006 / Accepted 11 April 2006

ABSTRACT

\begin{abstract}
We present results of a population synthesis study for semidetached short orbital period binaries which contain low-mass $\left(\lessgtr 1.5 M_{\odot}\right)$ donors and massive $\left(\gtrsim 4 M_{\odot}\right)$ compact accretors, which in our model represent black holes. Evolution of these binaries is determined by nuclear evolution of the donors and/or orbital angular momentum loss due to magnetic braking by the stellar wind of the donors and gravitational wave radiation. According to our model the estimated total number of this type of black-hole binaries in the Galaxy is $\gtrsim 10000$. If the magnetic braking removing angular momentum in semidetached black-hole binaries is described by the Verbunt \& Zwaan formula, the model predicts that among them around 3000 systems with periods $>2 \mathrm{~h}$ would be transient. In addition one finds several hundreds of luminous and stable systems with periods between 3 and $8 \mathrm{~h}$. Several dozens of these bright systems should be observed above the RXTE ASM sensitivity limit. The absence of such systems implies that upon Roche-lobe overflow by the low-mass donor angular momentum losses are reduced by a factor more than 2 with respect to the Verbunt \& Zwaan prescription. In such a case short period black-hole soft X-ray transients (SXT) may have donors that overflow the Roche lobe in the core hydrogen-burning stage. We show that it is unlikely that the transient behaviour of black-hole short-period X-ray binaries is explained by the evolved nature of the stellar companion. On the other hand a substantial fraction of black-hole binaries with periods $>3 \mathrm{~h}$ could be faint with truncated, stable cold accretion discs as proposed by Menou et al. Most of the semidetached black-hole binaries are expected to have periods shorter than $\sim 2 \mathrm{~h}$. Properties of such, still to be observed, very small mass-ratio $\left(q=M_{2} / M_{1}<0.02\right)$ binaries are different from those of their longer period cousins.
\end{abstract}

Key words. binaries: close - stars: evolution - X-rays: binaries

\section{Introduction}

All known low-mass X-ray binaries containing black hole components (massive compact accretors, hereafter LMBHB) are transient (McClintock \& Remillard 2005). The instability responsible for this, often intermittent, behaviour is apparently the same that drives dwarf-nova outbursts (see Lasota 2001, for a detailed review of the "disc instability model" - DIM) but in the case of LMBHB irradiation of the outer disc plays a crucial role (van Paradijs 1996; Dubus et al. 1999, 2001). For this instability to operate, rather low mass-transfer rates are required $\left(\lesssim 10^{-10}-10^{-9} M_{\odot} \mathrm{yr}^{-1}\right)$. This conjecture has led to the suggestion that all LMBHB are formed via a peculiar evolutionary path, in which the donor stars start mass transfer to the black hole only at the very end of their main-sequence lifetime (e.g. King et al. 1996; Ergma \& Fedorova 1998; Ergma \& Sarna 2001). It is therefore of interest to check which models of binary evolution are capable of reproducing the transient nature of the LMBHB population and their observed (or deduced from observations) mass-transfer rates. In addition population synthesis models can be used to test the statistics of the model versus the observations and the existence of a hidden (quasi)stable population of LMBHB postulated by Menou et al. (1999).
Among the 17 confirmed black hole binaries, (i.e. with a measured mass-function larger than $\sim 3 M_{\odot}$ ) ten are short-period $\left(P_{\text {orb }} \lesssim 1\right.$ day) systems (Orosz et al. 2004; McClintock \& Remillard 2005). Their evolution should be similar to that of cataclysmic variables (CV) which prompts to apply to LMBHB the model exploited for CV: evolution driven mainly by angular momentum loss via magnetically coupled stellar wind (MSW) and gravitational waves radiation (GWR). This is a non-trivial exercise because of the well-known problems of the "standard model" of CV evolution, e.g. the difficulty in reproducing the period gap and period minimum observed in the distribution of CVs (see e.g. Kolb 2002).

The criteria for the instability in the DIM depend mainly on the mass transfer rate, which is set by the period of the system and mass of the accreting body, i.e., along its evolutionary track a binary may move trough stable and unstable states.

Therefore the evolution of LMBHBs provides an interesting challenge to our understanding of both the stellar evolution and the accretion-disc physics. This prompted us to carry out a population synthesis study for low-mass binaries with massive companions based on a systematic investigation of the evolution of the latter systems under different assumptions on the angular 
momentum loss from the system. As the next step we attempted to infer the behaviour of a constructed population with respect to the stability criteria in DIM and to verify, whether it is possible that the Galaxy harbours a population of LMBHB with faint cold and stable discs. Population synthesis results are presented in Sect. 2 and compared to observations in Sect. 3. Discussion and conclusions follow.

\section{Formation of low-mass black-hole binaries and their current Galactic population}

\subsection{Formation of binaries containing a black hole and a low-mass star}

The formation of close binaries containing a black hole and a low-mass star is still not well understood. The progenitor of the black hole must have been a very massive star, with heavy mass loss, which went through a supernova explosion. It is remarkable that apparently a low-mass companion can survive such violent evolution. The "standard" formation channel for such binaries is an extension of the original formation scenario proposed for low-mass X-ray binaries with a neutron star accretor (van den Heuvel 1983): a massive star with a low-mass companion evolves and the low-mass star spirals into the giant in a common-envelope phase. The product of common-envelope stage is a compact binary consisting of the helium core of the massive star (a Wolf-Rayet star) and the low-mass companion. Neither the small amount of matter lost in supernova explosion in which the neutron star is formed nor a natal kick imparted to neutron star disrupt the system, so the binary survives and, due to angular momentum losses, becomes tighter and tighter until mass transfer ensues. In order to end with a black hole, one simply needs a more massive star to start with (e.g. McClintock \& Remillard 1986; de Kool et al. 1987). Unfortunately, the above scenario contains many very uncertain aspects: the amount of mass loss of massive stars, the outcome of the common-envelope phase, the amount of mass loss of the helium core after the common-envelope phase and the mass loss and possible asymmetric kick induced at the supernova explosion (see e.g. Langer 1989; Iben et al. 1995; Wellstein \& Langer 1999; Kalogera 1999; Fryer \& Kalogera 2001; Nelemans \& van den Heuvel 2001; Justham et al. 2006).

\subsection{Evolution of black-hole low-mass $X$-ray binaries: evolutionary computations}

In order to be able later to compare the properties of the observed black-hole low-mass X-ray binaries with our model, we have made detailed calculations of a grid of evolutionary sequences for different black hole and companion star masses and different initial periods. Depending on these parameters, the low-mass companions can overflow (if ever) their critical lobes at different evolutionary stages. Consideration of a range of initial periods is important, in particular to assess suggestion that transient lowmass black-hole X-ray binaries must all harbour donor-stars that almost exhausted hydrogen in their cores, e.g. (King et al. 1996; Ergma \& Fedorova 1998; Ergma \& Sarna 2001).

Evolutionary computations were carried out by means of the TWIN version of Eggleton (1971, priv. comm. 2003) evolutionary code. The most important updates of the code concerning equation of state, nuclear reaction network and opacity are described by Pols et al. (1995). Our version of the code implements angular momentum loss (AML) via a magnetically coupled stellar wind (MSW), following the model suggested by
Verbunt \& Zwaan (1981) and via gravitational wave radiation (GWR, Landau \& Lifshitz 1971). We should mention specifically that, at difference with other codes which include AML via MSW, it is assumed that a critical minimum depth of the convective zone $Z_{\text {conv }}>0.065 R_{\odot}$ is required for sustaining a significant surface magnetic field. Within assumptions used in the code, this limits the masses of main-sequence stars which may have MSW to lower than $1.6 M_{\odot}$. On the other hand, since it is believed that the magnetic field is anchored in the radiative core, MSW is switched-off as soon as convective envelope penetrates to $0.2 R_{\text {star }}$ ("disrupted" magnetic braking model). For initially unevolved MS-stars MSW terminates when their mass decreases to $\sim 0.3 M_{\odot}$.

The Verbunt \& Zwaan (1981) AML law can be written as

$\frac{\mathrm{d} J}{\mathrm{~d} t}=-0.510^{-28} f^{-2} k^{2} M_{\mathrm{d}} R_{\mathrm{d}}^{4} \omega^{3}$ dyn cm

where $k^{2} \sim 0.1$ is the gyration radius of the donor star and $M_{\mathrm{d}}$, $R_{\mathrm{d}}$ and $\omega$ are respectively its mass, radius and rotation angular frequency, $f$ is a parameter calibrating the strength of MSW AML. In the evolutionary code, the gyration radius is estimated for every model.

Solar chemical composition of the models was assumed. Trial computations for donors with metallicity $Z=0.008$ did not reveal any serious dependence of results on $Z$. Different metallicities might also influence the mass of the black hole, as the mass loss rates of massive stars are expected to scale with $Z^{0.5}$. However, what really matters for our purpose is the pre-SN mass. Since both mass loss rates for MS- and WR-stars are highly uncertain we neglect the Z-dependence of stellar winds. Comparison of our pre-SN masses with the results of trial computations with the Hurley et al. (2000) code which employs a different combination of stellar winds shows that pre-SN masses are the same within a few solar masses for metallicities of 0.02 and 0.008 . We neglected the possible influence of an irradiation induced stellar wind (Tavani \& London 1993) on the donor evolution. This is justified since irradiation feedback is important only for systems with $M \sim M_{\odot}$ donors (Büning \& Ritter 2005) which comprise a negligible minority of population under consideration.

In codes describing CV evolution the AML is usually normalized in a way to obtain the correct location of the period gap (see e.g. Kolb \& Willems 2005). Since no gap has been detected for the LMXB orbital distribution we left our code uncalibrated. Let us add that at variance with cataclysmic variables, in systems with massive primaries the donors are only slightly out of thermal equilibrium and the width of the calculated "period gap" is small, compared to CVs. For the models that overflow Roche lobes when they have already burnt a significant fraction of hydrogen in their cores the gap disappears (see Fig. 4 below).

The grids of evolutionary models were computed for initial secondary masses $\left(M_{20}\right)$ of $0.4-1.6 M_{\odot}$ and initial black hole masses $\left(M_{10}\right)$ of 4,7 , and $12 M_{\odot}$. In the first set of computations a "standard" model of evolution of low-mass binaries with AML via MSW ( $f=1$ and disrupted magnetic braking) and GWR was assumed (henceforth, model A). Since population synthesis under these "standard" assumptions did not provide satisfactory results (see below), in the second set of computations we assumed ad hoc that MSW does not operate in semidetached systems with black-hole components (henceforth, model B). In model B only GWR operates after RLOF. We also used for comparison a model similar to model A in which the MSW angularmomentum loss rate operated permanently, but reduced by a factor $\sim 2(f=1.37-$ model $\mathrm{C})$. Evolution models $\mathrm{A}, \mathrm{B}$, and $\mathrm{C}$ give 


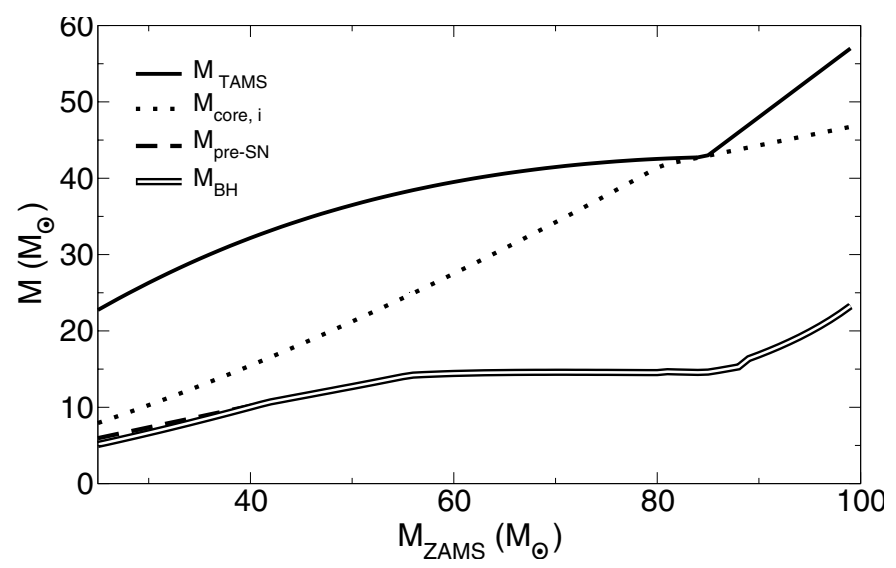

Fig. 1. Initial mass - final mass relations for massive stars in binaries. The lines show the mass of stars at the end of the main sequence (TAMS), the initial mass of helium core $M_{\text {core, }}$, the pre-supernova mass $M_{\text {pre-SN }}$, and the final black-hole mass $M_{\mathrm{BH}}$.

rise to 13737,12089 , and 11659 short-period semidetached systems, respectively. Corresponding LMBHB-population models are compared in detail in Sect. 3.

The models for low-mass donors typically ceased to converge at $M_{2}^{\min } \simeq 0.03 M_{\odot}$ in evolutionary tracks for models A and $\mathrm{C}$ and at $M_{2}^{\text {min }} \simeq 0.06 M_{\odot}$ for model $\mathrm{B}$. This happens because of the absence in the code of adequate tables of EOS and opacities for that low-mass stars (the slight difference in the limiting masses stems from some differences in the versions of the code used for models A and B, C; in our results this only slightly influenced $M_{2}^{\min }$ and position of orbital period minimum). For population modeling, all tracks were truncated at $M_{2}^{\min }=0.02 M_{\odot}$ or $0.06 M_{\odot}$, respectively. However, already at $M_{2}=0.06 M_{\odot}$ the mass ratio of components $q<0.02$, the circularization radius of accretion stream for such low $q$ becomes greater than the outer radius of the accretion disc and it is unclear, whether mass transfer happens at all (see 3.3 for more details). Thus, although evolutionary computations were continued beyond $q \approx 0.02$ with the same formalism as for larger $q$, the results may be not applicable to any physical systems.

\subsection{The Galactic population of low-mass binaries with black-hole companions}

We modeled the Galactic population of black-hole binaries using the population synthesis code SeBa (Portegies Zwart \& Verbunt 1996) with updates described in Portegies Zwart \& Yungelson (1998); Nelemans et al. (2001); Nelemans et al. (2004). The important points to note are that we assume a $50 \%$ binarity rate $(2 / 3$ of stars in binaries), an IMF after Kroupa et al. (1993), an initial distribution of separations in binary systems $(a)$ flat in $\log a$ between contact and $10^{6} R_{\odot}$, a flat mass ratio distribution, and initial distribution of eccentricities of orbits $\Xi(e)=2 e$.

A summary of the assumptions about the evolution of massive stars and Wolf-Rayet stars that we use in our code is shown in Fig. 1 where, for components of close binaries with initial masses of $M_{0}=25-100 M_{\odot}$, we show their masses at the end of the main sequence (TAMS), their initial helium core mass, their pre-supernova mass and the final black-hole mass ${ }^{1}$. We assume that the stellar wind mass-loss rate increases in time and

1 The overwhelming majority of close binaries donor stars lose their hydrogen envelope through mass transfer after the main sequence, but before the onset of helium core burning. For such binaries the final that in their total lifetime stars may lose an amount of matter that increases with initial mass: $0.01 M_{\mathrm{i}}^{2}$. If the star loses its hydrogen envelope, we switch to the Wolf-Rayet stellar mass-loss prescription. Mass loss on the main-sequence causes stars with masses of about $85 M_{\odot}$ to lose all their hydrogen before they reach the TAMS. For more massive stars the mass loss is so uncertain that we assume for simplicity that they lose $42 M_{\odot}$ on the main sequence, leading to the peculiar upturn of the solid line in Fig. 1. However, these extremely massive stars are so rare that they do not contribute to the population of low-mass black-hole binaries. For the common-envelope phase we use the standard prescription (Webbink 1984; de Kool et al. 1987), with the efficiency and structure parameters $\alpha$ and $\lambda$ combined: $\alpha \lambda=2$. For the mass loss by Wolf-Rayet stars formed after the commonenvelope phase, we use the law derived by Nelemans \& van den Heuvel (2001), which is based on the compilation of estimated mass-loss rates of observed Wolf-Rayet stars of Nugis \& Lamers (2000).

The formation of black holes in the code follows the fallback prescription of Fryer \& Kalogera (2001) whose basic assumption is that a constant fraction of the supernova explosion energy is used to unbind the envelope of the star. At difference to Fryer \& Kalogera (2001), who consider the explosion energy as a function of pre-supernova mass, we keep it fixed at $10^{50} \mathrm{erg}$, which is within the expected range but favours formation of rather massive black holes (up to $\sim 15 M_{\odot}$ ). For systems that are relevant for this study, i.e. naked helium cores, the prescription we use actually almost invariably results in complete collapse of the pre-supernova object: the CO core is very tightly bound while around this core only a small layer of helium is left because of the strong stellar wind during the preceding Wolf-Rayet phase. We assume that black holes receive a small asymmetric kick at formation (e.g. Jonker \& Nelemans 2004; Willems et al. 2005; Gualandris et al. 2005), which is taken from a Paczyński (1990) and Hartman (1997) velocity distribution with a dispersion of $300 \mathrm{~km} \mathrm{~s}^{-1}$, but scaled down with the ratio of the black hole mass to a neutron star mass. The population of short-period low-mass black-hole binaries is not sensitive to the assumed kick distribution, since scaled down with $M_{\mathrm{bh}} / M_{\mathrm{ns}}$-ratio kick velocities are too small to disrupt close binaries in SN explosions. This was confirmed by a test run assuming Maxwellian kick distribution for pulsars with $\sigma=265 \mathrm{~km} \mathrm{~s}^{-1}$ (Hobbs et al. 2005). The total number of systems that filled Roche lobe in Hubble time and evolved to shorter periods (in models A and B, see below) changed by several dozens only.

The model of the Galactic population of detached low-mass binaries with black-hole companions is then produced by folding the results of approximate evolutionary computations for a set of 250000 initial binaries with $M_{10} \geq 25 M_{\odot}$ with a time and position dependent model for the star formation history of the Galaxy that is based on results of Boissier \& Prantzos (1999) and a model for the distribution of stars in the Galaxy (see Nelemans et al. 2004). Some details of this model are described in Appendix A.

With our assumptions we find that, within a Hubble time (13.5 Gyr), 49000 binaries that have orbital periods below 2.0 day and contain black holes accompanied by main-sequence stars less massive than $1.6 M_{\odot}$ were formed in the Galaxy ${ }^{2}$. We restrict ourselves to $M_{20} \leq 1.6 M_{\odot}$ since for more massive

masses are rather insensitive to the initial period of the binary and the initial $q$.

2 This number is $\sim 0.01 \%$ of all binaries with black-hole components formed in Hubble time. 


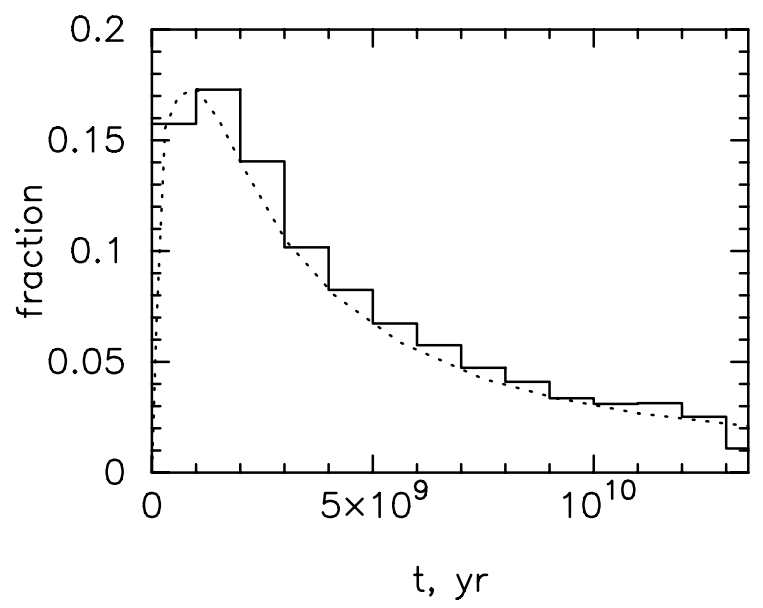

Fig. 2. Solid histogram - fractions of low-mass bh+ms binaries formed at different epochs in the lifetime of the Galaxy. Dotted curve shows the shape of star formation history adopted in the population synthesis code. At maximum, the star formation rate (SFR) is $\simeq 15.6 M_{\odot} \mathrm{yr}^{-1}$. Current SFR is $\simeq 1.9 M_{\odot} \mathrm{yr}^{-1}$.

stars magnetic braking is not supposed to operate. Out of these $\sim 49000$ binaries, $\sim 17000$ were brought into contact in a Hubble time, but only $\sim 14000$ evolved to shorter periods (Table 2). If only AML via GWR acts in systems with more massive donors, they experience case $\mathrm{AB}$ mass-transfer ${ }^{3}$, evolve into subgiants with increase of orbital periods and end their lives as helium white dwarfs.

Because black holes form in the first $~ 3$ Myr after their progenitor formation, the formation history of bh+ms binaries (Fig. 2) strictly follows the star formation history.

The upper panel of Fig. 3 shows the relation between initial masses of MS-components and orbital periods after circularization of the orbits after black hole birth events ${ }^{4}$. The lower panel represents relation between masses of components in these systems.

\subsection{The current Galactic population of black-hole low-mass $X$-ray binaries}

We have simulated the current Galactic population of semidetached low-mass black-hole binaries by convolving the above-described "underlying" population of binaries born at different epochs in the history of the Galaxy with the grids of pre-computed evolutionary tracks for low-mass components in binaries with different combinations of components and postcircularization (initial) orbital periods.

Since the features of the model population may be understood as a consequence of different patterns of evolution of lowmass donors (Fig. 4) we will first present examples of evolutionary tracks. The parameters of the tracks shown in Fig. 4 are listed in Table 1 . For every combination of $M_{20}$ and $M_{10}$ there are systems that, upon Roche lobe overflow (RLOF), evolve to shorter

3 According to common classification, in "case AB" mass-transfer starts when the donor is still in the core-hydrogen burning stage and continues in the hydrogen-shell burning stage.

${ }^{4}$ Because of specifics of time-realizations in the population synthesis code every dot in the plot actually represents four or five systems with similar combination of masses and orbital period, but born at different time. Since we present one random realization of the model for a population of black-hole+main-sequence star systems, all numbers given are subject to Poisson noise.
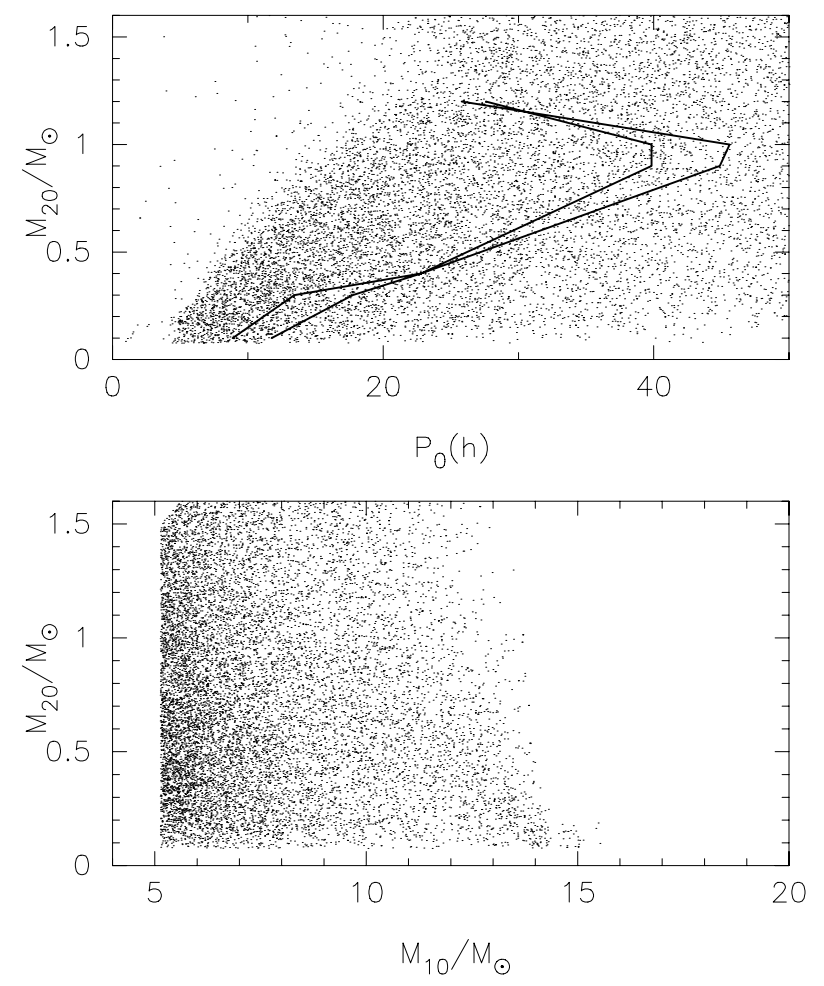

Fig. 3. Upper panel: relation between orbital periods and masses of main-sequence companions of black holes after circularization of the orbits. Systems with main-sequence components that may fill their Roche lobes in their main-sequence lifetime or in the Galactic disc lifetime (13.5 Gyr) are located to the left of the solid curves. Left and right solid curves correspond to $M_{\mathrm{BH}}=4 M_{\odot}$ and $12 M_{\odot}$. Lower panel: relation between initial masses of components in bh+ms systems.

periods having mass-transfer rates $\dot{M} \approx\left(10^{-9}-10^{-10}\right) M_{\odot} \mathrm{yr}^{-1}$; for $M_{20} \simeq 1 M_{\odot}$ this happens for systems with an abundance of hydrogen in their center $X_{\mathrm{c}} \gtrsim 0.05$ at RLOF. These systems may have post-circularization periods up to $\sim 40 \mathrm{~h}$ (Fig. 3) and they overflow Roche lobes at periods $\$ 12 \mathrm{~h}$ (see Table 1 and Fig. 4) since the period of semidetached systems depends only on the mass and radius of the donor and the latter changes only slightly in the hydrogen burning stage or practically does not change in Hubble time for $M_{20} \lesssim 0.95 M_{\odot}$. Systems with hydrogenexhausted cores (two rightmost tracks in Fig. 4, Nos. 7 and 8 in Table 1) evolve to longer periods. This is the well known effect of "bifurcation period" (Tutukov et al. 1985; Pylyser \& Savonije 1989). The bifurcation period $P_{\mathrm{b}}$ defines the right border of the position of new-born systems that evolve to shorter orbital periods upon RLOF, as shown in Fig. 4.

In systems that are initially driven by both MSW and GWR, $\dot{M}$ drops rapidly from $\dot{M} \sim 10^{-9} M_{\odot} \mathrm{yr}^{-1}$ to $\sim 10^{-10} M_{\odot} \mathrm{yr}^{-1}$ after termination of MSW. Like in cataclysmic variables, the evolution continues with decreasing $\dot{M}$ and there exists a minimum orbital period $\sim(70-80)$ min. LMBHB close to the period minimum have yet to be observed but our population synthesis model predicts that they might form the majority of LMBHB. These systems have very low mass ratios $q<0.02$ and it is far from obvious that mass transfer occurs in them in a similar fashion to standard compact semidetached binaries (see Sect. 3.3).

For the most massive stars that still have MSW and fill their Roche lobe close to $P_{\mathrm{b}}$, nuclear evolution continues to play a certain role after RLOF. As a result, they first evolve to shorter periods, but, when hydrogen is almost completely exhausted in their cores, turn to longer periods (tracks with "loops" in Fig. 4). 


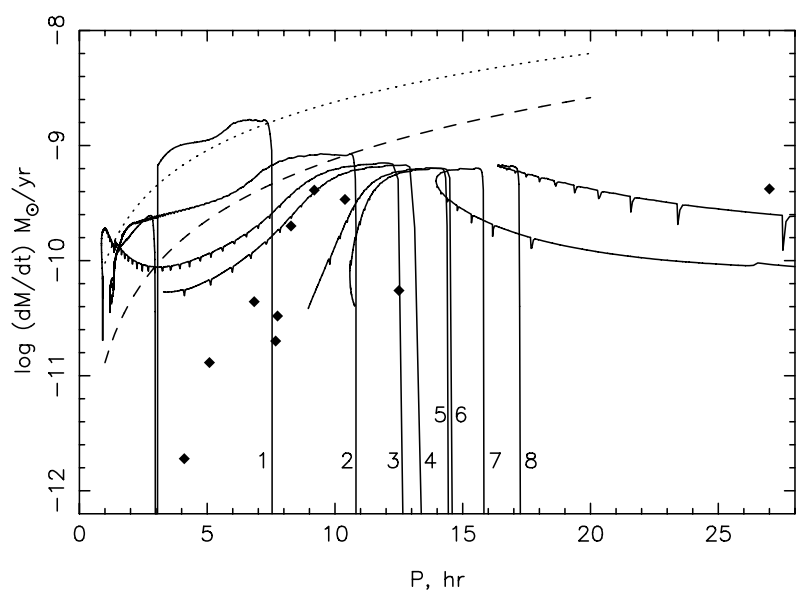

Fig. 4. Mass transfer rates vs. orbital period for systems with initial masses of components $1 M_{\odot}$ and $7 M_{\odot}$ that have different periods $P_{0}$ after circularization of orbits. Solid lines show tracks of donors evolving under "standard" assumption that AML via MSW operates until secondaries become convective $(f=1)$. The numbers along the tracks correspond to the numbers in Table 1 . The leftmost line is for $P_{0}=9.6 \mathrm{~h}$; in this system secondary fills its Roche almost unevolved at $P \approx 7.44 \mathrm{~h}$. Note the presence of a tiny period gap for this system. The systems to the right have $P_{0}$ from 38.4 to $45.6 \mathrm{~h}$ (respectively, at RLOF they have, either $X_{\mathrm{c}} \leq 7 \times 10^{-4}$ or helium core with $M_{\mathrm{He}}<0.01 M_{\odot}$, see Table 1). The dotted line shows mass transfer rate critical for disc instability (Eq. (2)). The dashed line marks the approximate upper limit to the average mass transfer rates for short-period black-hole binaries, as given by Eq. (8). The diamonds show estimates of mass-transfer rates based on assumed recurrence period, see Table 3.

Table 1. Parameters of tracks shown in Fig. 4. The columns list initial orbital period of the system, period at RLOF, central hydrogen abundance at RLOF, mass of helium core at RLOF.

\begin{tabular}{ccccc}
\hline \hline No. & $\begin{array}{c}P_{0}, \\
\text { hour }\end{array}$ & $\begin{array}{c}P_{\mathrm{c}}, \\
\text { hour }\end{array}$ & $X_{\mathrm{c}}$ & $\begin{array}{c}M_{\mathrm{He} 0}, \\
M_{\odot}\end{array}$ \\
\hline 1 & 9.60 & 7.44 & 0.697 & - \\
2 & 38.40 & 10.80 & 0.049 & - \\
3 & 40.80 & 12.48 & $7 \times 10^{-4}$ & - \\
4 & 41.52 & 12.96 & $2 \times 10^{-4}$ & - \\
5 & 42.96 & 14.40 & $2 \times 10^{-8}$ & - \\
6 & 43.20 & 14.40 & $2 \times 10^{-9}$ & - \\
7 & 44.40 & 16.08 & - & $4 \times 10^{-3}$ \\
8 & 45.60 & 17.28 & - & $1 \times 10^{-2}$ \\
\hline
\end{tabular}

In the extreme case of $M_{20}=1.6 M_{\odot}$ donor, this "u-turn" may happen at a period as short as $\sim 4 \mathrm{~h}$, but such systems with periods from a very narrow interval of initial $P_{\text {orb }}$ are extremely rare in the parental population.

\subsubsection{Evolved companions?}

The systems that have at RLOF $0.05 \gtrsim X_{\mathrm{c}} \gtrsim 0$ evolve to short periods and may have from the very beginning of the Roche-lobe overflow $\dot{M}<10^{-9} M_{\odot} \mathrm{yr}^{-1}$. In the period range $4-12 \mathrm{~h}$ they have rather low mass-transfer rates: $\dot{M} \sim 10^{-10}-10^{-11} M_{\odot} \mathrm{yr}^{-1}$ (Tutukov et al. 1985). They are represented by the tracks Nos. 3-6 (with initial periods $40.8-43.2 \mathrm{~h}$ and periods at contact $12.5-14.8 \mathrm{~h}$ ) in the centre of Fig. 4 . In the model population, they do not evolve to periods below about $4 \mathrm{~h}$, since they have to be already significantly evolved at the instant of RLOF which leaves them only a few Gyr of evolutionary lifetime in
Table 2. Break-down of black-hole candidate populations into subgroups according to accretion disc stability. Model A - "standard" evolution model with disrupted magnetic braking $(f=1)$, Model B - model without MSW in semidetached systems. Model C corresponds to AML by MSW reduced by a factor $2(f=1.37)$. The total number of systems in contact is indicated for each model. The population is then separated into systems with $q<0.02$ for which description in all evolutionary calculations may be inadequate (see Sect. 3.3) and systems with $q \geq 0.02$. For the latter, the systems are further broken down into bright persistent systems (DIM stable, Sect. 3.1) and transient systems. Some of the systems marked a "transient" could be cold, stable, truncated-disc systems (MNL stable, Sect. 3.2.2).

\begin{tabular}{lrll}
\hline \hline & Model A & Model B & Model C \\
\hline Total & 13737 & 12089 & 11659 \\
$q \geq 0.02$ & 3272 & 5074 & 2991 \\
\hline Hot stable & 367 & 0 & 107 \\
Transient & 2905 & 5074 & 2884 \\
(Cold stable) & $(62)$ & $(2495)$ & $(79)$ \\
\hline
\end{tabular}

semidetached state. Since the interval of periods of these systems and their mass-transfer rate $\dot{M}$ overlap with the those of SXT it is tempting to assume that they provide the explanation of the properties of these systems. However, this cannot be the case because in the majority of systems the donors fill their Roche lobes almost unevolved: for the systems shown in Fig. 4, the ratio of initial period intervals of systems that fill Roche lobes "evolved" and "unevolved" is $\sim 8 \%$. The actual ratio of the numbers of stars in two groups is much lower, since only systems with $M_{20} \gtrsim 0.95 M_{\odot}$ may exhaust hydrogen in their centres in Hubble time. This is confirmed by the upper panel of Fig. 5 (see below), which shows that even the upper estimates of the average mass-transfer rates in observed SXT hardly may be explained by the standard AML via MSW and GR model, because of low number of systems with desired initial parameters in the parental population and their short lifetime in the observed period range. Simply removing all systems that will fall in the "unevolved" class by fine-tuning the initial period distribution of bh $+m$ s is difficult: the most obvious way would be to change the common-envelope efficiency. However we tried this by repeating our calculations with $\alpha \lambda=0.5$ and 5 (the standard value in our model is 2), but the "unevolved" class still dominates. The number of black hole + low-mass MS-star systems formed in a Hubble time was, compared to "standard" model, 50\% lower and $15 \%$ higher respectively for these two calculations ${ }^{5}$.

This shows that the transient nature of the short period LMBHB most likely cannot be explained by the presence of strongly evolved main-sequence companions as proposed by e.g. King et al. (1996), Ergma \& Fedorova (1998), Ergma \& Sarna (2001).

\section{Accretion in the modeled LMBHB population and its observational consequences}

\subsection{Bright, persistent systems}

\subsubsection{Disc stability}

Figure 5 (upper and middle panels) shows the modeled population of semidetached low-mass binaries with assumed black hole accretors in "standard" model (A) with disrupted magnetic braking. In Fig. 5, only systems for which DIM is valid and stability analysis may be applied are plotted ( 3000 systems). The

\footnotetext{
${ }^{5}$ Respectively 24000 and 58000 systems.
} 


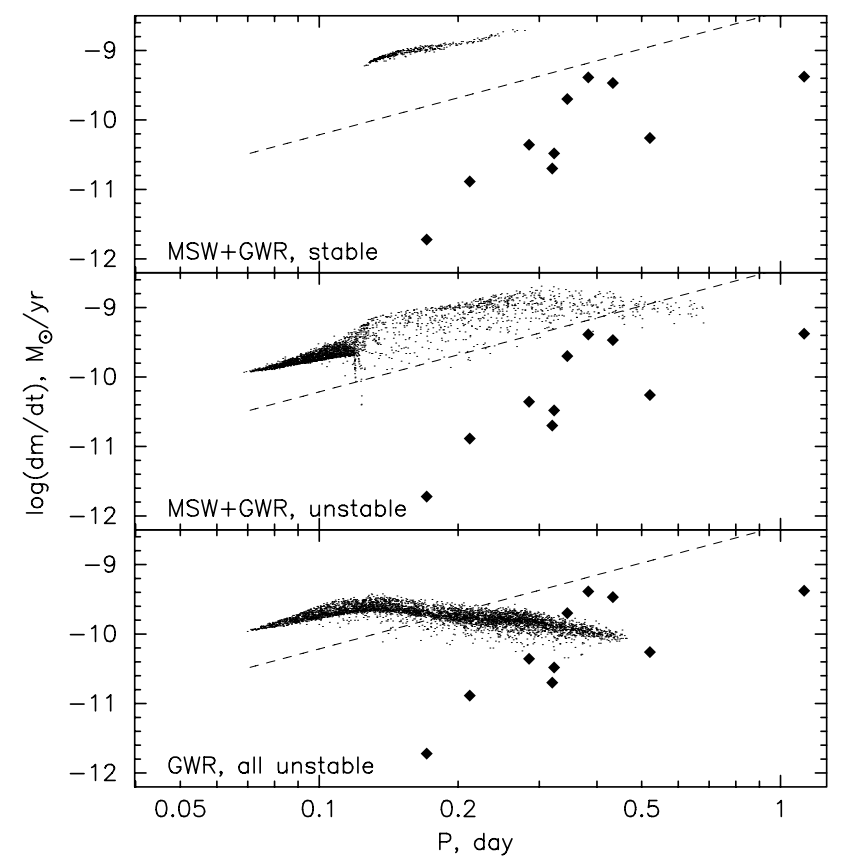

Fig. 5. Mass transfer rates vs. orbital period for the model population of semidetached low-mass binaries with assumed black hole accretors. Upper panel shows systems that have irradiated discs that are stable according to criterion given by Eq. (2) in the model for a "standard" assumption on AML via MSW always operating in stars with convective envelopes ( $f=1$, model A). Middle panel shows population of systems with unstable irradiated disks in the same model. Lower panel presents population for the model in which MSW does not operate in semidetached systems with black hole accretors (model B). In this model all binaries have unstable irradiated disks. The break-down over number of stable and unstable systems in particular models is listed in Table 2. The dashed line marks the approximate upper limit to the average masstransfer rates for short-period black-hole binaries, as given by Eq. (8), while diamonds show estimates of rates based on assumed recurrence period of 30 years, except for V616 Mon and IL Lup whose recurrence times are known to be $\sim 50$ and 10 years, respectively (see Table 3 and text for details).

remaining systems (Table 2) have low-mass ratios of components $(q<0.02)$ and low-mass $\left(M_{2}<0.03 M_{\odot}\right)$ donors. If mass transfer occurs and can be described by the "standard" model of evolution driven by AML by GWR, these systems should be concentrated around a "period minimum" at 70-80 min and beyond it and at mass-transfer rates $10^{-10}-10^{-11} M_{\odot} \mathrm{yr}^{-1}$. Model A contains $\sim 13000$ systems (Table 2 ) of which some may be luminous and non-transient (upper panel of Fig. 5). Such steady systems should satisfy the stability criterion according to which irradiated discs are stable if their accretion rate exceeds certain critical value $\dot{M}_{\text {crit }}^{+}$(van Paradijs 1996). According to Dubus et al. (1999) the latter is

$\dot{M}_{\text {crit }}^{+} \approx 2.4 \times 10^{-11} M_{\mathrm{BH}}^{-0.4}\left(\frac{R_{\mathrm{d}}}{10^{10} \mathrm{~cm}}\right)^{2.1}\left(\frac{C}{5 \times 10^{-4}}\right)^{-0.5} M_{\odot} \mathrm{yr}^{-1}$,

where $M_{\mathrm{BH}}$ is the mass of accretor in $M_{\odot}, R_{\mathrm{d}}$ is the disc outer radius, and $C$ is a measure of heating of the disc by X-rays (Shakura \& Sunyaev 1973); for a given disc radius $R$ the irradiation temperature $T_{\text {irr }}$ is

$T_{\text {irr }}^{4}=C \frac{\dot{M} c^{2}}{4 \pi \sigma R^{2}}$,

where $\sigma$ is the Stefan-Boltzmann constant. We use $C=5 \times 10^{-4}$, following Dubus et al. (1999) who have found that this value is consistent with properties of persistent low-mass X-ray sources. One should, however, keep in mind that $C$ does not have to be a constant and might e.g. vary in time (Esin et al. 2000).

The disc outer radius $R_{\mathrm{d}}$, which depends only on the mass ratio once scaled to the orbital separation, is estimated using Table 2 of Paczyński (1977) who computed the largest possible stable, periodic non-intersecting orbits. This estimate is very close to the one obtained by Papaloizou \& Pringle (1977) by studying the linear response to tidal forcing. For mass ratios $q \approx 0.02$, the disc outer radius becomes comparable to the circularization radius $R_{\text {circ }}$ and the $R_{\mathrm{d}}$ estimate is not valid. It is then uncertain how accretion proceeds. In what follows we concentrate on systems with mass ratios $q \geq 0.02$ (3272 systems in model A), corresponding to periods longer than $\gtrsim 2 \mathrm{~h}$, and come back to the systems with low mass ratios in Sect. 3.3.

\subsubsection{Excess of luminous persistent LMBHB}

The number of systems for each model population is given in Table 2, which also gives the number of luminous, persistent systems expected in each case according to the criterion (2).

The consistency of these numbers can be compared to observations, which show no persistent LMBHB with short $P_{\text {orb }}$. Possible exceptions, i.e. systems showing variability that could be different from what is called a transient behaviour, such as GX 339-4 or GRS 1915+105 have long orbital periods and are not addressed here. Model A (standard MSW) predicts the highest number of persistent sources. These 367 systems have an average luminosity $L=0.1 \dot{M} c^{2} \approx 5 \times 10^{36} \mathrm{erg} \mathrm{s}^{-1}$. Given their location in the Galaxy provided by the population synthesis code, we estimate the intervening absorption assuming that the gas is, roughly, distributed as $n=1.8 \exp (-d / 3.5 \mathrm{kpc}-$ $z / 0.125 \mathrm{pc}) \mathrm{cm}^{-3}$ with $d$ the distance to the Galactic centre, $z$ the height above Galactic plane and where the gas is distributed like the thin disk population (Bahcall 1986) with a density and scale-height taken from Zombeck (1990). The average column density along the line of sight from the Sun (at $8.5 \mathrm{kpc}$ from the centre) to the systems is $\log N_{\mathrm{H}} \approx 22.1$.

The accretion luminosity may be assumed to be emitted as that of a $1 \mathrm{keV}$ blackbody, corresponding to the "high/soft" X-ray spectral state of LMBHB. Taking into account X-ray absorption, the number of persistent systems expected above a threshold of $7 \times 10^{-11} \mathrm{erg} \mathrm{s}^{-1} \mathrm{~cm}^{-2}$ in the $2-10 \mathrm{keV}$ band is 85 . This threshold corresponds to the completeness limit for the X-ray sources seen by the RXTE All Sky Monitor (about $3 \mathrm{mCrab}$ or 0.2 counts/s) deduced from the $\log N-\log S$ distribution of extragalactic sources by Grimm et al. (2002). Setting the threshold ten times higher still yields 13 bright, persistent LMBHB with ASM fluxes comparable to e.g. SMC X-1.

Assuming a power-law spectrum with photon index -2 (flat in $v F_{v}$ ), corresponding to the "low/hard" X-ray spectral state of LMBHB, one obtains almost the same number of detectable persistent systems: a $1 \mathrm{keV}$ blackbody peaks at $2.7 \mathrm{keV}$ so the power-law emission is not much different. Persistent LMBHB might escape detection if they emit most of their energy below $1 \mathrm{keV}$. Indeed, KV UMa in the low/hard state had blackbodylike emission peaking at $\approx 25 \mathrm{eV}$, with a spectral luminosity almost 10 times greater than in X-rays where it displays a power law spectrum (McClintock et al. 2001). Assuming that only 10\% of the accretion luminosity is emitted in hard X-rays would still imply 16 persistent systems above $3 \mathrm{mCrab}$ in the RXTE ASM (1 above $30 \mathrm{mCrab}$ ). Alternatively, the discs may be not irradiated, in which case no systems would be stable; this, however, would be surprising as both persistent NS LMXBSs and 
outbursting black hole transients show clear signatures of irradiation. Note that with parameter $C=5 \times 10^{-4}$ (Eq. (3)), only a very moderate $0.5 \%$ of the accretion luminosity (efficiency of $10 \%)$ is reprocessed in the disc.

Despite obvious uncertainties, the large number of systems at high mass-transfer rates in Model A makes it difficult to avoid the presence of a significant number of observed persistent systems. However, mass-transfer rates could be smaller than suggested by models of binary evolution driven by AML through MSW and GWR. In such a case Model B (GWR only) predicts no persistent systems at all while the intermediate case represented by Model C (reduced MSW) yields 29 systems above $3 \mathrm{mCrab}$ (or 5 above $30 \mathrm{mCrab}$ ) in the RXTE ASM. In addition mass-transfer rates predicted by model $\mathrm{A}$ are too large compared to observations of transient systems. These points are further discussed in the next section.

\subsubsection{Reduced magnetic braking}

While energy and angular momentum loss via gravitational wave radiation is beyond doubt, the issue of angular momentum loss by binaries via magnetically coupled stellar wind and its quantitative description are much more arguable. The earliest formulation of Eq. (1) by Verbunt \& Zwaan (1981) and its variations are based on extrapolation of stellar rotation braking law (Skumanich 1972) over several orders of magnitude in stellar rotation rates, imply efficient spin-orbit coupling for low-mass star and has been modified and/or challenged by many authors (Hameury et al. 1988; Collier Cameron 2002; Andronov et al. 2003; Ivanova \& Taam 2003, and others); Baraffe \& Kolb (2000) argued that in order to explain the cataclysmic binary period distribution (i.e. their evolution) a magnetic braking law according to which the mass-transfer rate would, contrary to the "standard" case, decrease with increasing orbital period might be necessary. It is therefore fair to conclude that the so-called "magnetic braking mechanism" is so uncertain that it can be almost modified at will.

For this reason we computed a grid of evolutionary sequences for binaries with low-mass donors and massive accretors under the assumption that AML via MSW does not operate in semidetached $b h+m s$ systems. This choice was motivated by studies such as Andronov et al. (2003), Ivanova \& Taam (2003) according to which the MSW operating in close binaries is reduced by at least one order of magnitude with respect to Verbunt \& Zwaan (1981) law; but mass-transfer values obtained in this way should be rather treated as lower limits. Based on this set of evolutionary tracks we derived the second model for the population of semidetached binaries with black holes (model B). The model is presented in the lower panel of Fig. 5. In this model the current Galactic population of semidetached bh+ms binaries contains in total, $\approx 12000$ objects, about 5000 of which have $q \geq 0.02$ (Table 2).

The absence of MSW AML results in lower $\dot{M}$ in the systems with similar initial masses of components and postcircularization periods. As a result, in all systems of model $\mathrm{B}$ accretion discs are unstable according to the criterion (2). Instability limit is given by the lower bound of region filled by stable systems in "standard model". In a model without magnetic braking in semidetached systems typical mass-transfer rates $\dot{M}$ are by factor up to 10 lower.

If MSW were to be absent from the very beginning, before the RLOF, the results would not change much. The number of systems would be reduced by a factor $\sim 2$. However, the longest
Table 3. Orbital periods, mass-transfer rates based on recurrence times and upper limits on the average mass-transfer rate for black-hole candidate short-period binaries. See text for details.

\begin{tabular}{lrrc}
\hline \hline Name & $\begin{array}{r}P_{\text {orb }}, \\
\text { day }\end{array}$ & $\begin{array}{r}\left\langle\dot{M}_{\text {recc }}\right\rangle, \\
M_{\odot} \mathrm{yr}^{-1}\end{array}$ & $\begin{array}{c}\dot{M}_{\text {in }}, \\
M_{\odot} \mathrm{yr}^{-1}\end{array}$ \\
\hline XTE J1118+480 (KV UMa) & 0.171 & $1.9 \times 10^{-12}$ & $1.5 \times 10^{-10}$ \\
GRO J0422+32 (V518 Per) & 0.212 & $1.3 \times 10^{-11}$ & $2.3 \times 10^{-10}$ \\
GRS 1009-45 (MM Vel) & 0.285 & $4.4 \times 10^{-11}$ & $3.8 \times 10^{-10}$ \\
XTE J1650-500 & 0.320 & $2.0 \times 10^{-11}$ & $4.8 \times 10^{-10}$ \\
A0620-00 (V616 Mon) & 0.323 & $3.3 \times 10^{-11}$ & $4.8 \times 10^{-10}$ \\
GS 2000+25 (QZ Vul) & 0.345 & $2.0 \times 10^{-10}$ & $5.4 \times 10^{-10}$ \\
XTE J1859+226 (V406 Vul) & 0.383 & $4.1 \times 10^{-10}$ & $6.4 \times 10^{-10}$ \\
GRS 1124-68 (GU Mus) & 0.433 & $3.4 \times 10^{-10}$ & $8.0 \times 10^{-10}$ \\
H 1705-25 (V2107 Oph) & 0.521 & $5.5 \times 10^{-11}$ & $1.1 \times 10^{-9}$ \\
4U 1543-47 (IL Lup) & 1.125 & $4.2 \times 10^{-10}$ & $4.3 \times 10^{-9}$ \\
\hline
\end{tabular}

period systems would be absent and this would make consistency with observations worse.

The absence of stable bright low-mass sources argues in favour of an AML reduced as compared to predictions of Verbunt \& Zwaan (1981) magnetic braking model. It is worth noting that Menou et al. (1999) also found that black-hole SXTs evolution should be driven by AML without MSW. In the view of both theoretical (the magnetic braking law, the value of $C$ ) and observational (the number of luminous, stable but undetected systems) uncertainties it would not make much sense to try to fix the value of parameter $f$ in Eq. (1) that would bring the calculated population in exact agreement with observations. An experiment with MSW angular momentum loss rate reduced by factor 2 (Model C, see Table 2) shows that finding such an agreement will require a more substantial reduction of the magnetic braking AML.

\subsection{Transient systems and cold, stable systems}

The prediction of our model can also be compared to masstransfer rates deduced from observations of SXT sources. Of course such deductions are notoriously uncertain but, as we argue below, they can provide interesting information especially when combined with the knowledge that all observed shortperiod LMBHB are transient X-ray systems.

\subsubsection{Reservoir discs}

The usual method for SXT consists of obtaining the masstransfer rate by dividing the mass accreted during outburst by the recurrence time (see e.g. White \& van Paradijs 1996). Regretfully, among short-period SXT the recurrence time is known only for A0620-00 (about 60 years) and 4U 1543-47 (about 10 years). For the other systems one can only obtain upper limits by assuming that since only one outburst has been seen the recurrence time is longer than 30 years. These estimates of $\dot{M}$, derived from the data on outburst parameters from Chen et al. (1997) and for KV UMa and V406 Vul based of observations presented in Chaty et al. (2003) and Hynes et al. (2002) respectively, are given in the penultimate column of Table 3 and are shown in Figs. 4-6.

Clearly even pure GWR cannot explain such low masstransfer rates if one assumes that they represent secular values. No LMBHB with a period $\gtrsim 4 \mathrm{~h}$ can have a secular mass-transfer rate as low as $\dot{M} \sim 10^{-12} M_{\odot} \mathrm{yr}^{-1}$; it would take post-minimum period systems several Hubble times to reach these periods and 


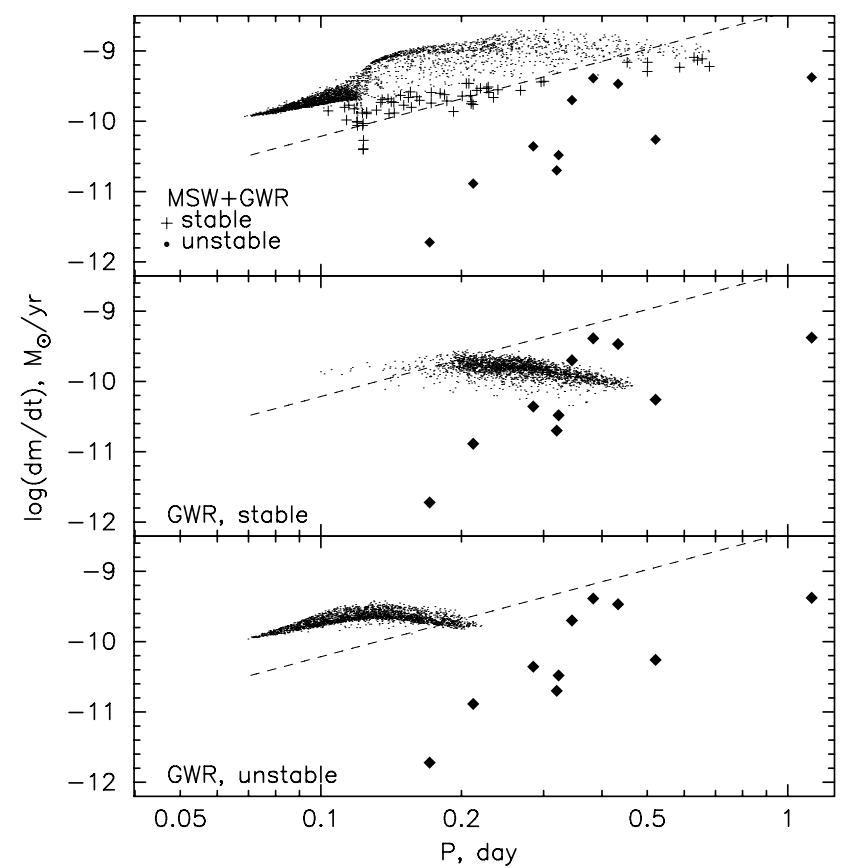

Fig. 6. Mass transfer rates vs. orbital period for the model population of semidetached low-mass binaries with assumed black hole accretors. Upper panel - "standard" evolution model with disrupted magnetic braking braking (model A). Pluses mark stable according to Menou et al. stability criterion for cold discs systems, while dots - unstable ones. Middle panel shows position of systems with discs stable acording to Menou et al. criterion in the model in which MSW does not operate in semidetached systems with black hole accretors (model B). Lower panel - systems with unstable discs in model B. Diamonds are mass-transfer rates estimated on the base of assumed recurrence periods, like in Fig. 5.

corresponding mass-transfer rates. The only known type of systems where such $\dot{M}$ may be expected, are binaries with initially degenerate dwarf donors, but even then orbital periods should be $\lesssim 2 \mathrm{~h}$ (Deloye et al. 2005). If the extremely low mass-transfer rate deduced for short period SXTs were due to downward fluctuations from an intrinsically high secular mass-transfer rate, one would still have to explain why the bright counterparts are not observed (Sect. 3.1). Furthermore, some systems would then necessarily have higher rates than their secular value (by how much depends on the duty cycle of the purposed low states), hence there should be even more persistent systems.

For longer periods, Fig. 4 shows, $\dot{M}$ of systems with $P_{\text {orb }} \approx$ 8-10 h may be consistent with evolution of systems where donors overflow Roche lobes extremely close to TAMS, but then one needs to assume some very special initial distribution of binaries over orbital separations which will finally, after several evolutionary stages with mass transfer and mass and momentum loss from the system and supernova explosion, result in concentration of zero-age black-hole and main-sequence-star systems just at the desired very narrow range of separations.

The longest period SXT of the "short-period" group, $4 \mathrm{U} 1543-47$ is, in principle, consistent with the evolution of systems with donors overflowing Roche-lobes after formation of He-cores. But again, these binaries evolve through the Hertzsprung gap extremely fast, and the probability of observing a descendant of such systems is very low, see discussion of intermediate-mass "Hertzsprung gap" black-hole binaries in Kolb (1998).

\subsubsection{Truncated discs}

It is, however, plausible that the mass-transfer rates in shortperiod SXTs are much higher than those derived above. The method used to obtain the low values mentioned above treats the disc as a reservoir which during quiescence is filled up to a critical value at which the outburst is triggered and the disc emptied. The implicit assumption in this picture is that the reservoir is not leaky, i.e. that the accretion rate at the disc's inner edge is much smaller than the mass-transfer rate, as indeed predicted by the DIM when the disc extends to the innermost stable circular orbit. However, there are many reasons to think that quiescent (and not only quiescent, see e.g. Done \& Gierliński 2006) SXT discs are truncated and therefore "leaky" because of the inner truncation required to explain observed quiescent luminosities (e.g. Lasota 1996; Lasota et al. 1996). Also, Dubus et al. (2001) showed that the disc instability model can reproduce the observed X-ray light-curves only if discs in SXTs are truncated (and irradiated). The truncated disc "paradigm" is now commonly used in describing the observed timing and spectral properties of X-ray LMBHB (McClintock \& Remillard 2005, and references therein).

For systems with non-stationary quiescent accretion discs the mass-transfer rate can be estimated as (Lasota 2001)

$\dot{M}_{\mathrm{tr}} \approx \frac{\epsilon M_{\mathrm{D}, \max }}{t_{\mathrm{recc}}}+\dot{M}_{\mathrm{in}}$

where

$M_{\mathrm{D}, \max }=2.7 \times 10^{21} \alpha^{-0.83}\left(\frac{M_{1}}{M_{\odot}}\right)^{-0.38}\left(\frac{R_{\mathrm{d}}}{10^{10} \mathrm{~cm}}\right)^{3.14} \mathrm{~g}$

is the maximum quiescent-disc mass and $\epsilon=\Delta M_{\mathrm{D}} / M_{\mathrm{D} \text {, max }}$ the fraction of the disc's mass lost during outburst; $\alpha$ is the kinematic viscosity parameter, $R_{\mathrm{d}}$ is the disc's outer radius and $\dot{M}_{\text {in }}$ is the accretion rate at the disc's inner edge. The usual, non-leaky disc estimates, neglect $\dot{M}_{\text {in }}$. For a stable equilibrium disc $t_{\text {recc }}=\infty$ and $\dot{M}_{\text {tr }}=\dot{M}_{\text {in }}$.

However, in SXTs $\dot{M}_{\text {in }}$ is in fact the dominant term as argued by Menou et al. (1999). They applied models in which the inner part of the accretion flow was represented by an ADAF (Narayan \& Yi 1994; Abramowicz et al. 1995) to several quiescent SXT systems. Fitting the model to the observed spectra provided estimates of $\dot{M}_{\text {in }}=\dot{M}_{\mathrm{ADAF}}$ which are higher than the "refilling" term $\epsilon M_{\mathrm{D}, \max } / t_{\text {recc }}$, for which except for A0620-00 (and the long period SXT system V404 Cyg) only upper limits could be obtained.

Lasota (2000) suggested a general estimate of $\dot{M}_{\text {in }}$ in quiescent SXTs. It is based on the fact that in quiescence according to the DIM the disc accretion rate has to satisfy the inequality:

$\dot{M}(r)<\dot{M}_{\text {crit }}^{-} \approx 6.4 \times 10^{-11}\left(\frac{M_{1}}{M_{\odot}}\right)^{-0.88}\left(\frac{r}{10^{10} \mathrm{~cm}}\right)^{2.65} M_{\odot} \mathrm{yr}^{-1}$

(Dubus 1998; Lasota 2001, note that this criterion which requires the whole quiescent disc to be in a cold thermal equilibrium state is different from the instability condition of Eq. (2)). Therefore, the mass accretion rate at the truncation radius $\left(\dot{M}_{\text {in }}\right)$ must be smaller than $\dot{M}_{\text {crit }}^{-}\left(r_{\text {in }}\right)$. Inside the truncation radius the mass accretion rate $\dot{M}$ is constant $\left(\dot{M}=\dot{M}_{\text {in }}\right)$, the accretion timescale in the ADAF (or similar type of flow) being very short. Parameterizing the truncation radius as a fraction of the circularization radius (Frank et al. 2002) $r_{\text {in }}=f_{\mathrm{t}} r_{\text {circ }}$ (where $f_{\mathrm{t}}<0.48$, 
see Menou et al. 1999) and assuming a mass ratio $q \approx 0.1$ one obtains

$\dot{M}_{\text {in }} \lesssim 10^{-8} f_{\mathrm{t}}^{2.65} P_{\text {day }}^{1.77} M_{\odot} \mathrm{yr}^{-1}$,

which because of the assumed value of $q$ is independent of the primary's mass. Taking $f_{\mathrm{t}} \approx 0.48$ one obtains

$\dot{M}_{\text {in }} \lesssim 3.6 \times 10^{-9} P_{\text {day }}^{1.77} M_{\odot} \mathrm{yr}^{-1}$

which, considering the uncertainties, corresponds reasonably well to the observed $L_{\mathrm{X}}\left(P_{\text {orb }}\right)$ relation for quiescent SXTs (Hameury et al. 2003), or at least can be considered as an upper limit.

Using Eq. (8) one obtains upper limits to mass-transfer rates given in the last column of Table 3 . They are consistent with the model B and one can see that a small amount of additional AML could provide an even better agreement. One can therefore conclude that a substantially reduced MSW AML would be consistent with both the stability properties and mass-transfer rates of SXTs.

\subsubsection{Number of transients}

Estimates of the number of SXTs in our Galaxy based on observations range from several hundreds to a couple thousand (van den Heuvel 1992; Chen et al. 1997; Romani 1998). The number of SXTs predicted by the models is 3000 (Model A) to 5000 (Model B), on the upper end of the estimates based on observations. Assuming that their outbursts peak on average at $0.1 L_{\text {edd }}$ in X-rays, all of these transients should be seen in outburst by the RXTE ASM, regardless of their location in the Galaxy. The current discovery rate seems to be $\approx 2-5$ per year. Matching this rate would imply recurrence times of several hundred years. Chen et al. (1997) find that the average peak luminosity of transients is $\log L \approx 37.7 \pm 0.8$. The peak flux could be overestimated: with a peak at $10^{37} \mathrm{erg} \mathrm{s}^{-1}$ in the $R X T E$ $A S M$ bandpass, only $\approx$ half of the systems are above a $30 \mathrm{mCrab}$ threshold (appropriate for transients) after absorption, yielding recurrence rates closer to $\approx 100$ years. Given the relatively high number of transients expected, the population synthesis taken at face value would suggest that many transients are sub-Eddington at maximum and/or that recurrence times are $\gtrsim 100$ years for most sources.

\subsubsection{A population of faint LMBHB?}

A model by Menou et al. (1999) (MNL) may be the answer to this apparent difficulty. Following a suggestion by Lasota et al. (1996) they pointed out that if the truncation radii were slightly larger than estimated to fit observations of quiescent SXTs the discs would be globally stable. Indeed, stationary $(\dot{M}=$ const.) discs truncated at

$R_{\text {in }}>R_{\text {crit }}=1.69 \times 10^{9}\left(\frac{\dot{M}}{10^{-10} M_{\odot}}\right)^{0.375}\left(\frac{M_{1}}{M_{\odot}}\right)^{0.33}$

are cold and globally stable since their accretion rates are lower than the critical value given by Eq. (6) corresponding to cold stable equilibria. Hence, some of the systems classified by us as transients would instead be cold, stably accreting sources. Menou et al. (1999) suggested that a population of such persistent LMBHB could be present in the Galaxy. Despite their relatively large mass-transfer rates $\left(\dot{M} \sim 10^{-10} M_{\odot} \mathrm{yr}^{-1}\right)$ these systems would be very faint and difficult to detect so it is interesting to establish if their putative existence is compatible with population models.

Figure 6 shows the distribution of stars in two population models with respect to stability criterion suggested by MNL. Here, we assume the truncation occurs at $R_{\text {in }}=0.48 R_{\text {circ }}$, the radius at which overflowing material from the donor star merges with the accretion disc (Menou et al. 1999). MNL-stable systems have $R_{\text {in }}>R_{\text {crit }}$. The break-down over MNL-stable and unstable (transient) systems is given in Table 2. In the "standard" case with MSW AML (model A) very few discs that were unstable vs. DIM-criterion, become stable vs. MNL-criterion. On the other hand, about a half of DIM unstable systems become stable vs. the MNL criterion in the model without MSW after RLOF (model B). This is because mass transfer rates in the GWR-only scenario are much lower than in the MSW+GWR scenario, thereby enabling the criterion for a cold disc to be met more easily. The number of transients for both models stays at $\approx 2000-3000$. On the other hand, truncating the disc at $R_{\text {circ }}$ would make all transients "MNL-stable" for both models. It is therefore a distinct possibility that there are very few "classical" SXTs in the DIM sense (i.e. regularly outbursting) and that the LMBHB population divides up into hot/stable and cold/stable systems, the latter erupting from time to time due to weather variability in truncation physics or mass transfer rate. Even in this extreme case, the predicted population size is too small to have cold/stable systems contribute significantly to the large number of faint $\left(L_{\mathrm{X}} \sim 10^{31} \mathrm{erg} \mathrm{s}^{-1}\right)$ hard X-ray sources seen in deep Chandra exposures toward the Galactic Center (Muno et al. 2003; Belczynski \& Taam 2004).

\subsection{Systems with very low mass ratios}

Mass transfer in systems with very low mass ratios is uncertain and they have been left out of the discussion until now. For values of $q \lesssim 0.02$ the circularization radius becomes greater than the estimates of the outer radius given by Paczyński (1977) and Papaloizou \& Pringle (1977). Matter flowing in from the companion circularises onto unstable orbits. At $q \approx 0.02$, matter is added at $R_{\text {circ }}$ onto orbits that can become eccentric due to the $3: 1$ resonance. At $q \approx 0.005$ the circularization radius approaches the 2:1 Lindblad resonance. This can efficiently prevent mass being transferred onto the compact object (by analogy with the gaps created by planets in protoplanetary discs or the inner edges of circumbinary discs) unless the timescale of mass transfer is such that the gap can be filled before it is cleared (unlikely, as the condition for gap formation is roughly $M_{2} / M_{\odot} \gtrsim t_{\text {dyn }} / t_{\text {vis }}$, where $t_{\text {dyn }}$ and $t_{\text {vis }}$ are respectively the dynamical and viscous timescales). Subsequent evolution is probably set by the balance between GW radiation (dominating at low q) which drives the system inward and the torque exerted by the disc which drives the system outward. Regardless of this exact balance, since the outer radius of the accretion disc is unlikely to be greater than $R_{\text {circ }}$, most of these systems (if accreting) are likely to be MNL stable according to the discussion in the preceding section.

\section{Discussion and conclusions}

We have modeled populations of short-period semi-detached close binary systems containing black holes and low-mass stars under various assumptions about the AML mechanism. In all cases the obtained population can be divided into two subsets. One, contains short-period ( $\leqslant 0.1$ day), very low mass-ratio 
( $q<0.02)$ systems; the other contains systems whose orbital parameters correspond to those determined for black hole binary transients. Mass transfer in short-period systems may be interrupted by resonances within the primary's Roche lobe and their subsequent evolution and the observational consequences are uncertain. For the systems with mass ratios $q>0.02$, the disk instability model provides a framework to investigate the observational consequences in terms of bright, persistent systems and transients.

Among systems with the range of periods encountered in SXTs (let us note that the shortest observed period of a LMBHB is $4.1 \mathrm{~h}$ but nothing in our model prevents such systems to have periods $\sim 2 \mathrm{~h}$ ) the number of transient systems depends on the assumed AML mechanism. The standard Verbunt \& Zwaan (1981) formula implies the presence of luminous stable systems that are not observed. On the other hand, AML driven solely by GWR yields only transient systems. This suggests that in short-period LMBHB the strength of the AML MSW mechanism is significantly reduced. Such a reduction is also required by the values of mass-transfer rates deduced from observations. Our population model shows that the required mass-transfer rates cannot be due to the evolved nature of the mass-donor.

The number of transients in the models is in the few thousand range, at the upper end of estimates based on observations. Better agreement would imply transients having lower peak fluxes and/or long recurrence times. Another possibility is that the number of transients is reduced compared to these estimates. A significant fraction of transients could be cold and stable if the thin accretion disc is truncated in quiescence. The number of such systems is highest with GWR-only angular momentum losses (lower mass transfer rates): with $R_{\text {trunc }}=0.48 R_{\text {circ }}$, roughly half of transient SXTs then become cold and stable. With MSW+GWR, the number of cold, stable systems is very small. This fraction can be increased to include all systems if the disc is truncated up to $R_{\text {circ }}$. If this were the case, transients would be flukes (not triggered by the DIM but by external factors) and the regularly outbursting SXTs envisioned by the DIM would be an exception rather than the rule as suggested by Lasota et al. (1996).

Recently, Podsiadlowski et al. (2003) and Justham et al. (2006) suggested that low-mass companion stars are not likely to provide sufficient gravitational potential to unbind during common-envelope phase the envelope of black-hole's massive progenitor. In our formulation, this is equivalent to a low value of the product of common-envelope efficiency and structure parameter $\alpha \lambda$. A trial computation with $\alpha \lambda=0.1$ resulted in a population with only $\simeq 3400$ short-period binaries containing black holes with low-mass companions. Only the most massive binaries avoided merger due to the fact that they lose more mass on the main sequence and thus have rather low-mass envelopes and survive the common-envelope phase. However, all black holes produced in this case have mass exceeding $\simeq 14 M_{\odot}$, substantially higher than what is observed in short-period LMBHB. Postcircularization period - secondary mass relations for these binaries are similar to these shown in Fig. 3. Thus, if such systems were formed, we expect evolution similar to described in this paper, but without formation of LMBHB resembling observed ones. However, it is difficult to reach firm conclusions on this issue as both the details of the common-envelope phase as well as the formation of black holes are very uncertain. So low-mass stars may still be able to remove the envelope or if indeed only the most massive binaries survive, their black hole masses may be lower and be consistent with the observed masses.
We conclude that modeling the population of LMBHB, using standard models of stellar evolution and accretion physics, leads to prediction of (1) a significant population of systems with $q<0.02$ and $P_{\text {orb }} \leq 2 \mathrm{~h}$; (2) a significant number of persistent LMBHB unless MSW angular momentum losses are reduced; (3) a high number of transients unless the peak X-ray fluxes are low and/or the recurrence time high; (4) a possibility of having a large fraction, if not all LMBHB systems, actually being (secularly) cold and stable.

Acknowledgements. We thank Peter Eggleton for providing the copy of his evolutionary code and Samuel Boissier for providing the table of star formation rates and helpful discussion about the Galactic model. GN is supported by PPARC and NWO. LRY is supported by NWO, NOVA, RFBR, and Russian Academy of Sciences Basic Research Program "Origin and Evolution of Stars and Galaxies". LRY acknowledges warm hospitality and support from Institut d'Astrophysique de Paris, Université Pierre et Marie Curie, and Astronomical Institute "Anton Pannekoek", where a part of this study was carried out. GD, JPL and LRY were supported by grants from the Centre National d'Études Spatiales and the GDR PCHE of the CNRS. SPZ acknowledges support from KNAW and LKBF.

Note added in proof: under assumptions accepted in the present paper it is possible that massive stars with low-mass companions avoid merger in the common envelope (CE), in disagreement with Podsiadlowski et al. (2003). The possibility of merger avoidance sensitively depends on the parameters of stellar massloss, time-dependence of stellar radii and CE evolution. Massloss in late stages of evolution is highly uncertain and $\mathrm{CE}$ treatment based on simple energy balance is a forced simplification. We consider our assumptions reasonable for the moment and hope that the problem will be resolved when more observational data on mass-loss will become available and the processes in common-envelopes better understood.

\section{Appendix A: Model for the star formation history and stellar density distribution}

The probability of a star being born at a radius $R$ or smaller depends on the integrated $S F R$, i.e.,

$P(R, t)=\frac{\int_{0}^{R} \operatorname{SFR}\left(R^{\prime}, t\right) 2 \pi R^{\prime} \mathrm{d} R^{\prime}}{\int_{0}^{R_{\max }} \operatorname{SFR}\left(R^{\prime}, t\right) 2 \pi R^{\prime} \mathrm{d} R^{\prime}}$,

where $R_{\max }$ is the maximum extent of the Galactic disc (19 kpc). We assume that the stars do not migrate radially in time. The age of the Galaxy in this model is 13.5 Gyr. Since the Boissier \& Prantzos (1999) model gives the SFR projected onto the plane of the Galaxy, we assume a $z$-distribution in the Galactic disc

$P(z) \propto \operatorname{sech}\left(z / z_{\mathrm{h}}\right)^{2}$,

where $z_{\mathrm{h}}=200 \mathrm{pc}$, neglecting dependence on age and mass. We have added a bulge to the model of Galactic distribution of stars, by doubling the SFR in the inner $3 \mathrm{kpc}$ of the Galaxy compared to Boissier \& Prantzos (1999). The total mass in this region at the age of $13.5 \mathrm{Gyr}$ is then $2.6 \times 10^{10} M_{\odot}$, consistent with kinematic and micro-lensing results (e.g., Klypin et al. 2002). We distribute the stars in the bulge according to

$\rho_{\text {bulge }}=\exp \left(-(r / 0.5 \mathrm{kpc})^{2}\right)$

where $r=\sqrt{x^{2}+y^{2}+z^{2}}$. The time-dependence of SFR under given assumptions and comparison to often used exponential SFR may be found in Fig. 2 of Nelemans et al. (2004). 


\section{References}

Abramowicz, M. A., Chen, X., Kato, S., Lasota, J.-P., \& Regev, O. 1995, ApJ, 438, L37

Andronov, N., Pinsonneault, M., \& Sills, A. 2003, ApJ, 582, 358

Bahcall, J. N. 1986, ARA\&A, 24, 577

Baraffe, I., \& Kolb, U. 2000, MNRAS, 318, 354

Belczynski, K., \& Taam, R. E. 2004, ApJ, 616, 1159

Boissier, S., \& Prantzos, N. 1999, MNRAS, 307, 857

Büning, A., \& Ritter, H. 2005, in The Astrophysics of Cataclysmic Variables and Related Objects, ASP Conf. Ser., 330, 61

Chaty, S., Haswell, C. A., Malzac, J., et al. 2003, MNRAS, 346, 689

Chen, W., Shrader, C. R., \& Livio, M. 1997, ApJ, 491, 312

Collier Cameron, A. 2002, in The Physics of Cataclysmic Variables and Related Objects, ASP Conf. Ser., 261, 11

de Kool, M., van den Heuvel, E. P. J., \& Pylyser, E. 1987, A\&A, 183, 47

Deloye, C. J., Bildsten, L., \& Nelemans, G. 2005, ApJ, 624, 934

Done, C., \& Gierliński, M. 2006, MNRAS, 121

Dubus, G. 1998, Ph.D. Thesis

Dubus, G., Hameury, J.-M., \& Lasota, J.-P. 2001, A\&A, 373, 251

Dubus, G., Lasota, J., Hameury, J., \& Charles, P. 1999, MNRAS, 303, 139

Eggleton, P. P. 1971, MNRAS, 151, 351

Ergma, E., \& Fedorova, A. 1998, A\&A, 338, 69

Ergma, E., \& Sarna, M. J. 2001, A\&A, 374, 195

Esin, A. A., Lasota, J.-P., \& Hynes, R. I. 2000, A\&A, 354, 987

Frank, J., King, A., \& Raine, D. J. 2002, Accretion Power in Astrophysics: Third Edition (Cambridge University Press), 398

Fryer, C. L., \& Kalogera, V. 2001, ApJ, 554, 548

Grimm, H.-J., Gilfanov, M., \& Sunyaev, R. 2002, A\&A, 391, 923

Gualandris, A., Colpi, M., Portegies Zwart, S. F., \& Possenti, A. 2005, ApJ, 618

Hameury, J.-M., Barret, D., Lasota, J.-P., et al. 2003, A\&A, 399, 631

Hameury, J. M., Lasota, J. P., King, A. R., \& Ritter, H. 1988, MNRAS, 231, 535

Hartman, J. W. 1997, A\&A, 322, 127

Hobbs, G., Lorimer, D. R., Lyne, A. G., \& Kramer, M. 2005, MNRAS, 360, 974

Hurley, J. R., Pols, O. R., \& Tout, C. A. 2000, MNRAS, 315, 543

Hynes, R. I., Haswell, C. A., Chaty, S., Shrader, C. R., \& Cui, W. 2002, MNRAS, 331,169

Iben, I. J., Tutukov, A. V., \& Yungelson, L. R. 1995, ApJS, 100, 233

Ivanova, N., \& Taam, R. E. 2003, ApJ, 599, 516

Jonker, P. G., \& Nelemans, G. 2004, MNRAS, 354, 355

Justham, S., Rappaport, S., \& Podsiadlowski, P. 2006, MNRAS, 88

Kalogera, V. 1999, ApJ, 521, 723

King, A. R., Kolb, U., \& Burderi, L. 1996, ApJ, 464, L127

Klypin, A., Zhao, H., \& Somerville, R. S. 2002, ApJ, 573, 597

Kolb, U. 1998, MNRAS, 297, 419

Kolb, U. 2002, in ASP Conf. Ser. 261: The Physics of Cataclysmic Variables and Related Objects, 180

Kolb, U., \& Willems, B. 2005, in The Astrophysics of Cataclysmic Variables and Related Objects, ASP Conf. Ser., 330, 17

Kroupa, P., Tout, C. A., \& Gilmore, G. 1993, MNRAS, 262, 545
Landau, L. D., \& Lifshitz, E. M. 1971, Classical theory of fields, 3rd edn. (Oxford: Pergamon)

Langer, N. 1989, A\&A, 220, 135

Lasota, J. P. 1996, in Compact Stars in Binaries, IAU Symp., 165, 43

Lasota, J.-P. 2000, A\&A, 360, 575

Lasota, J.-P. 2001, New Astron. Rev., 45, 449

Lasota, J.-P., Narayan, R., \& Yi, I. 1996, A\&A, 314, 813

McClintock, J., \& Remillard, R. 2005, in Compact Stellar X-ray Sources, ed. W. Lewin, \& M. van der Klis (Cambridge: CUP), in press [arXiv: astro-ph/0306213]

McClintock, J. E., Haswell, C. A., Garcia, M. R., et al. 2001, ApJ, 555, 477

McClintock, J. E., \& Remillard, R. A. 1986, ApJ, 308, 110

Menou, K., Narayan, R., \& Lasota, J. 1999, ApJ, 513, 811

Muno, M. P., Baganoff, F. K., Bautz, M. W., et al. 2003, ApJ, 589, 225

Narayan, R., \& Yi, I. 1994, ApJ, 428, L13

Nelemans, G., \& van den Heuvel, E. P. J. 2001, A\&A, 376, 950

Nelemans, G., Yungelson, L. R., \& Portegies Zwart, S. F. 2004, MNRAS, 349, 181

Nelemans, G., Yungelson, L. R., Portegies Zwart, S. F., \& Verbunt, F. 2001, A\&A, 365, 491

Nugis, T., \& Lamers, H. J. G. L. M. 2000, A\&A, 360, 227

Orosz, J. A., McClintock, J. E., Remillard, R. A., \& Corbel, S. 2004, ApJ, 616, 376

Paczyński, B. 1977, ApJ, 216, 822

Paczyński, B. 1990, ApJ, 348, 485

Papaloizou, J., \& Pringle, J. E. 1977, MNRAS, 181, 441

Podsiadlowski, P., Rappaport, S., \& Han, Z. 2003, MNRAS, 341, 385

Pols, O. R., Tout, C. A., Eggleton, P. P., \& Han, Z. 1995, MNRAS, 274, 964

Portegies Zwart, S. F., \& Verbunt, F. 1996, A\&A, 309, 179

Portegies Zwart, S. F., \& Yungelson, L. R. 1998, A\&A, 332, 173

Pylyser, E. H. P., \& Savonije, G. J. 1989, A\&A, 208, 52

Romani, R. W. 1998, A\&A, 333, 583

Shakura, N. I., \& Sunyaev, R. A. 1973, A\&A, 24, 337

Skumanich, A. 1972, ApJ, 171, 565

Tavani, M., \& London, R. 1993, ApJ, 410, 281

Tutukov, A. V., Fedorova, A. V., Ergma, E. V., \& Yungelson, L. R. 1985, SvAL, 11,52

van den Heuvel, E. P. J. 1983, in Accretion-driven stellar X-ray sources, ed.

W. H. G. Lewin, \& E. P. J. van den Heuvel (Cambridge: CUP), 303

van den Heuvel, E. P. J. 1992, Endpoints of stellar evolution: The incidence of stellar mass black holes in the galaxy, Tech. Rep.

van Paradijs, J. 1996, ApJ, 464, L139

Verbunt, F., \& Zwaan, C. 1981, A\&A, 100, L7

Webbink, R. F. 1984, ApJ, 277, 355

Wellstein, S., \& Langer, N. 1999, A\&A, 350, 148

White, N. E., \& van Paradijs, J. 1996, ApJ, 473, L25

Willems, B., Henninger, M., Levin, T., et al. 2005, ApJ, 625, 324

Zombeck, M. V. 1990, Handbook of space astronomy and astrophysics, 2nd edn. (Cambridge: University Press) 